www.nature.com/ejhg

\title{
ARTICLE
}

\section{WFS1 mutations in Spanish patients with diabetes mellitus and deafness}

\author{
Enric Domènech ${ }^{1}$, Montse Gómez-Zaera ${ }^{1}$ and Virginia Nunes ${ }^{\star, 1}$ \\ ${ }^{1}$ Centre de Genètica Mèdica i Molecular, Institut de Recerca Oncologica (I.R.O.) Gran Via s/n Km 2,7, 08907 \\ L'Hospitalet de Llobregat, Barcelona, Spain
}

\begin{abstract}
Wolfram syndrome (WS) is an autosomal recessive neurodegenerative disorder characterised by early onset diabetes mellitus and progressive optic atrophy, as well as other clinical features such as deafness, diabetes insipida, renal tract abnormalities and diverse psychiatric illnesses. A gene responsible for WS was identified in 4p16.1 (WFS1). It encodes a putative 890 amino acid transmembrane protein expressed in a wide spectrum of tissues. Recently, a new locus for WS has been located on 4q22-24, providing additional evidence for the genetic heterogeneity of this syndrome. We have studied the presence of WFS1 variants in three groups of individuals: patients with diabetes mellitus, patients with deafness and patients with both conditions. A fourth group of healthy subjects was used as control. We have identified a total of 18 nucleotide changes in the WFS1 gene: three mutations and 15 polymorphisms. Six of these changes were previously undescribed. Four of the 15 polymorphisms studied among the patients group present statistical differences in the allelic and genotypic distribution when comparing affected vs control groups.
\end{abstract}

European Journal of Human Genetics (2002) 10, 421 -426. doi:10.1038/sj.ejhg.5200823

Keywords: Wolfram syndrome; WFS1; diabetes mellitus; deafness; mutation; polymorphism

\section{Introduction}

Wolfram syndrome (WS, MIM 222300, also named DIDMOAD, the acronym of diabetes insipida, diabetes mellitus, optic atrophy and deafness) is a rare autosomal recessive neurodegenerative disease. WS patients typically develop diabetes mellitus in the first decade of life, followed by deafness and bilateral optic atrophy in the second decade of life. ${ }^{1}$ Other clinical features such as psychiatric disorders, renal tract abnormalities, diabetes insipida or peripheral neuropathy have been described among WS patients. $^{2-4}$

Linkage between WS phenotype and a marker located in the short arm of chromosome 4 was demonstrated by Polymeropoulos et $\mathrm{al}^{5}$ and later, in 1998, a gene responsi-

*Correspondence: Dra. Virginia Nunes; Centre de Genètica Mèdica i Molecular. Institut de Recerca Oncològica (I.R.O.). Hospital Duran i Reynals. Gran Via s/n Km 2,7. 08907 L'Hospitalet de Llobregat, Barcelona, Spain; Tel: 93/260.74.27; Fax: 93/260.74.14; E-mail: vnunes@iro.es Received 31 January 2002; revised 8 April 2002; accepted 17 April 2002 ble for WS named WFS1, was identified on $4 \mathrm{p} 16.1 .^{6,7}$ WFS1 encodes a putative 890 amino acid protein named by some groups wolframin, with no functional homology with any known family of proteins. Biochemical studies and immunofluorescent cell staining showed that wolframin is an endoglycosidase H-sensitive membrane glycoprotein that localizes primarily in the endoplasmic reticulum. ${ }^{8}$

Recently, linkage analysis has identified an additional locus for WS in the region 4q22-24. ${ }^{9}$ Phenotypically, the WS patients in that study do not show diabetes insipida and some of them suffer gastrointestinal ulceration, proving once more the genetic and phenotypic complexity of this syndrome. ${ }^{9,10}$

We wanted to ascertain the presence of WFS1 gene mutations in patients with diabetes mellitus and/or neurosensorial deafness, since these two conditions are characteristic of WS patients. Mutations in one of the two copies of the WFS1 gene in an individual could be contri- 
buting to the development of diabetes mellitus, deafness or other symptoms described in WS patients, but not the full syndrome.

A large number of variants in the WFS1 gene has been reported previously. ${ }^{4}$ Considering this, the main objective of this study is to identify mutations in the WFS1 gene in a Spanish population affected with diabetes mellitus and/ or neurosensorial deafness. We will also analyse to what extent these changes could contribute to the phenotype studied.

\section{Materials and methods}

Patients and controls

Individuals studied in this work belonged to four categories:

(1) Twenty-three unrelated subjects affected of type 2 diabetes mellitus and neurosensorial deafness (DM+F) (10 men and 13 women, range: $29-74$ years).

(2) Forty-eight unrelated subjects with autosomal recessive deafness (F) (24 men and 24 women, range: 4-83 years).

(3) Thirty-eight type 2 diabetes mellitus patients (DM) (20 men and 18 women, range: $42-81$ years).

(4) Forty-nine healthy unrelated controls also participated in this work (24 males and 25 females, range: 26-61 years). None of them had previous history of diabetes mellitus or deafness.

\section{Analysis of WFS1 gene}

DNA was extracted from peripheral blood samples obtained after informed consent following standard procedures.

The WFS1 gene has eight exons. In this study we screened exons 2 to 8 , the latter being subdivided into seven regions 8-1 to 8-7, following previous works. ${ }^{7}$ The thirteen pairs of primers and conditions for the PCRs are reported elsewhere. ${ }^{7}$ After PCR, SSCP analysis was performed, we ran premade $12.5 \%$ acrylamide SSCPs gels (ExcelGel DNA analysis kit, Pharmacia Biotech, Sweden) at $5^{\circ} \mathrm{C}$. A 20 -min prerun at $100 \mathrm{~V}, 23 \mathrm{~mA}$ and $5 \mathrm{~W}$ was performed, followed by a 3-h 20-min run at $600 \mathrm{~V}, 30 \mathrm{~mA}$ and $18 \mathrm{~W}$. Silver staining of these gels was completed with the DNA Silver Staining kit on an automatic gel stainer (Pharmacia Biotech).

Sequencing reactions were carried out with the same primers used in the previous PCRs. The sequencing reaction mix contained primers, forward and reverse for each sample at a final concentration of $0.3 \mu \mathrm{M}, 2 \mu \mathrm{l}$ of Big Dye Terminator Cycle Sequencing ready Reaction Kit (Applied Biosystems, USA), $2 \mu$ l of purified PCR product (QIAquick Gel Extraction Kit, Qiagen, Germany) and $3 \mu$ l of distilled water, in a final volume of $10 \mu \mathrm{l}$.

Sequencing PCR consisted of an initial denaturation step at $94^{\circ} \mathrm{C}$ for $5 \mathrm{~min}, 30$ cycles of denaturing at $94^{\circ} \mathrm{C}$ for $30 \mathrm{~s}$, annealing at $50^{\circ} \mathrm{C}$ for $15 \mathrm{~s}$ and a long extension at $60^{\circ} \mathrm{C}$ for
4 min, then a final extension at $60^{\circ} \mathrm{C}$ for $10 \mathrm{~min}$. PCR products were purified through G-50 columns (Pharmacia Biotech) and loaded in an ABI Prism 377 sequencer (Applied Biosystems). Sequences were then analysed using the programme Sequencher v 3.0 (Gene Codes Corporation, USA).

The sequence variants are considered mutations when they imply an amino acid change and are not found in controls. Other variants not included in this category are considered polymorphisms.

\section{Statistical analysis}

Hardy-Weinberg equilibrium of the different genotypes was evaluated for each polymorphism by Chi-squared with Jurg Ott's HWE-program version 1.5. Control genotypes follow H-W equilibrium (data not shown), so we can assume no stratification for our population and panmixy. Statistical significance of differences between distributions for alleles and genotypes was evaluated using Fisher's exact test with a $2 \times 2$ or $3 \times 2$ contingency table, respectively, comparing the three patient groups $v s$ control group. Significance was considered when $P<0.05$. Yule's coefficient was evaluated for each polymorphism with statistical differences in its allelic distribution, in order to ponder the intensity of association.

\section{Results \\ WFS1 mutations}

Two new mutations, as well as a previously described one, were found in the WFS1 gene among DM+F and F patients. Figure 1 shows the heterozygotic pattern of these mutations. Changes 2185G $>\mathrm{A}$ and $2269 \mathrm{C}>\mathrm{A}$, both on exon 86 , were found in two unrelated $\mathrm{DM}+\mathrm{F}$ patients, each in a different subject. These mutations cause the amino acid changes D729N and L757I, respectively. Mutation D729N represents the change from a charged amino acid to a polar one, whereas L757I mutation keeps the aliphatic characteristic of the amino acid replaced. The third mutation $2611 \mathrm{G}>\mathrm{A},{ }^{11}$ in exon $8-7$, encodes for the V871M amino acid change. It represents the change of a small aliphatic amino acid to a hydrophobic. This variation has been found in a DM+F subject and in a deaf patient (a 74 year old man and a 15 year old woman, respectively). The segregation of the mutation V871M among the family of the deaf patient was then studied. The mutation was found in heterozygosis in her 14 year old deaf sister and her father, a non affected 37 year old man.

\section{Allelic frequencies of the polymorphisms}

We have analysed the frequencies of alleles of the polymorphisms found in the four groups studied. Fifteen polymorphisms have been identified, four of them previously unreported: T346T, L432V, Y455Y and V813A (see Table 1). Significant statistical differences in the allelic distribution of polymorphisms V395V $(P=0.0002), \mathrm{H} 611 \mathrm{R}$ $(P<0.0001)$, K811K $(P<0.0001)$ and S855S $(P<0.0001)$ were 
A

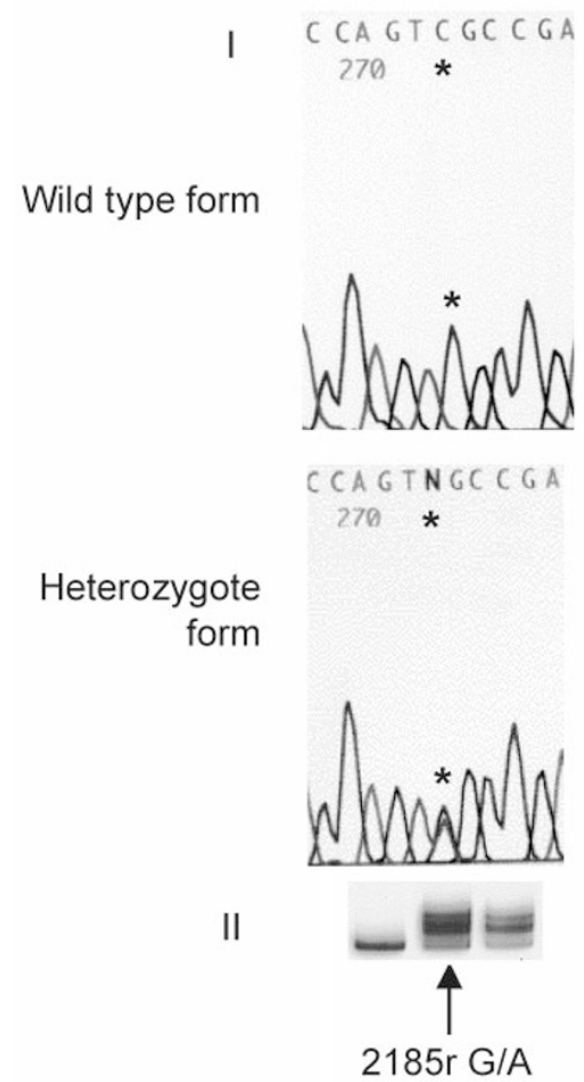

B
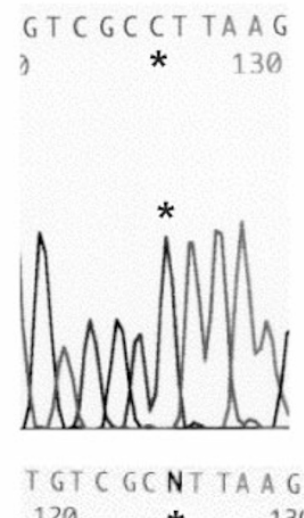

120

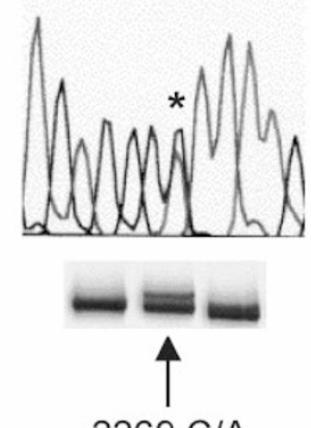

2269 C/A
C

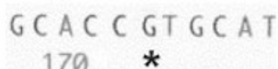

$170 *$
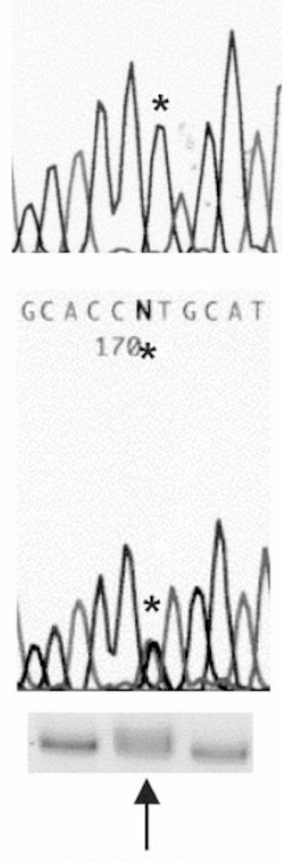

2611G/A

Figure 1 Electropherograms of the three mutations found in the WFS1 gene. (A) and (B) Correspond to exon 8-6, whereas C to exon 87. Electropherogram $A$ is shown as the reverse sequence. * Indicates the mutated nucleotide. II: SSCP pattern of these three mutations. Arrows indicates the heterozygous SSCP pattern.

found in the DM+F group compared to control group (Table 1). In addition, there were significant statistical differences in the allelic distribution of polymorphisms $\mathrm{K} 811 \mathrm{~K}$ $(P=0.035)$ and S855S $(P<0.0001)$, among the DM and control groups (Table 1). No significant statistical differences in the allelic distribution of any polymorphism in the $\mathrm{F}$ group has been found (Table 1).

Yule's coefficient was evaluated for those alleles with significant statistical distribution, revealing low intensity of association (data not shown).

\section{Genotypic frequencies of the polymorphisms}

Significant statistical differences in the distribution of genotypes of polymorphisms V395V $(P=0.0004)$, H611R $(P<0.0001)$, K811K $(P=0.0002)$ and S855S $(P=0.0006)$ were found when comparing $\mathrm{DM}+\mathrm{F}$ vs control groups (Table 1). The DM group showed significant statistical differences for H611R $(P=0.016)$ and S855S $(P<0.0001)$ polymorphisms in their genotypic frequencies, when comparing with the control group. No significant differences were found for the F group (Table 1).

\section{Discussion}

We have identified three mutations in heterozygosis (Figure 1) in three patients affected with type 2 diabetes mellitus and deafness. These are amino acid substitutions that conserve the main biochemical properties. According to the predicted structure of the wolframin, ${ }^{4}$ the mutations found are located in the intra-cytoplasmatic domain, near to the C-Terminal end. The lack of knowledge about the function of WFS1 makes it difficult to explain the possible contribution of these mutations to the above mentioned diseases.

Mutations D729N and L751I were found in the DM+F group. At this moment, we can not postulate with which disease, diabetes mellitus or deafness, these mutations could be related. A study of the familial transmission for these mutations in the families of affected subjects may help to clarify this point.

Mutation V871M, detected in a $\mathrm{DM}+\mathrm{F}$ subject, was also found in heterozygosis in two deaf sisters and in her unaffected father. By studying the frequencies of alleles and genotypes of the WFS1 polymorphisms in the four groups 
Table 1 Allelic and genotypic frequencies of the polymorphisms found in WFS1 gene

\begin{tabular}{|c|c|c|c|c|c|c|c|c|c|c|c|c|}
\hline$\frac{\text { Region }}{8-1}$ & \multicolumn{2}{|l|}{ Polymorphism } & \multicolumn{4}{|c|}{ Frequencies of alleles } & \multicolumn{5}{|c|}{ Frequencies of genotypes } & \\
\hline & C. $n=48 t$ & 72 & 0.75 & 24 & 0.25 & & 25 & 0.52 & 22 & 0.46 & 1 & 0.02 \\
\hline & $\mathrm{DM}+\mathrm{F}, n=23$ & 40 & 0.87 & 6 & 0.13 & 0.45 & 17 & 0.74 & 6 & 0.26 & 0 & 0 \\
\hline & $\mathrm{F}, n=46$ & 72 & 0.78 & 20 & 0.22 & 0.83 & 26 & 0.57 & 20 & 0.43 & 0 & 0 \\
\hline $8-1$ & $1023 \mathrm{C}>\mathrm{T} ; \mathrm{F} 341 \mathrm{~F}$ & $\mathrm{C}$ & & $\mathrm{T}$ & & & $\mathrm{C} / \mathrm{C}$ & & $\mathrm{C} / \mathrm{T}$ & & $\mathrm{T} / \mathrm{T}$ & \\
\hline & C, $n=48 t$ & 89 & 0.93 & 7 & 0.07 & & 41 & 0.85 & 7 & 0.15 & 0 & 0 \\
\hline $8-2$ & $185 \mathrm{~T}>\mathrm{C} ; \mathrm{V} 395 \mathrm{~V}$ & $\mathrm{~T}$ & & $\mathrm{C}$ & & & $\mathrm{T} / \mathrm{T}$ & & $\mathrm{T} / \mathrm{C}$ & & $\mathrm{C} / \mathrm{C}$ & \\
\hline & C, $n=49$ & 71 & 0.72 & 27 & 0.28 & & 23 & 0.47 & 25 & 0.51 & 1 & 0.02 \\
\hline & $\mathrm{DM}+\mathrm{F}, n=23^{*}$ & 45 & 0.98 & 1 & 0.02 & 0.06 & 22 & 0.96 & 1 & 0.04 & 0 & 0 \\
\hline & $\mathrm{DM}, n=32$ & 39 & 0.61 & 25 & 0.39 & 1.68 & 10 & 0.31 & 19 & 0.59 & 3 & 0.1 \\
\hline & $\mathrm{F}, n=47$ & 68 & 0.72 & 26 & 0.28 & 1.00 & 22 & 0.47 & 24 & 0.51 & 1 & 0.02 \\
\hline $8-2$ & $1294 C>C ; L 432 V$ & $\mathrm{C}$ & & G & & & $\mathrm{C} / \mathrm{C}$ & & $\mathrm{C} / \mathrm{G}$ & & G/G & \\
\hline & C, $n=49$ & 96 & 0.98 & 2 & 0.02 & & 47 & 0.96 & 2 & 0.04 & 0 & 0 \\
\hline & $\mathrm{DM}+\mathrm{F}, n=23$ & 46 & 1 & 0 & 0 & 0.42 & 23 & 1 & 0 & 0 & 0 & 0 \\
\hline & $\mathrm{DM}, n=32$ & 63 & 0.98 & 1 & 0.02 & 0.76 & 31 & 0.97 & 1 & 0.03 & 0 & 0 \\
\hline & F, $n=47$ & 91 & 0.97 & 3 & 0.03 & 1.58 & 45 & 0.96 & 1 & 0.02 & 1 & 0.02 \\
\hline $8-2$ & $1364 C>T ; Y 455 Y$ & $\mathrm{C}$ & & $T$ & & & $\mathrm{C} / \mathrm{C}$ & & $\mathrm{C} / \mathrm{T}$ & & $\mathrm{T} / \mathrm{T}$ & \\
\hline & C, $n=49$ & 98 & 1 & 0 & 0 & & 49 & 1 & 0 & 0 & 0 & 0 \\
\hline & $\mathrm{DM}+\mathrm{F}, n=23$ & 46 & 1 & 0 & 0 & & 23 & 1 & 0 & 0 & 0 & 0 \\
\hline & $\mathrm{DM}, n=32$ & 64 & 1 & 0 & 0 & & 32 & 1 & 0 & 0 & 0 & 0 \\
\hline & F, $n=47$ & 93 & 0.99 & 1 & 0.01 & 3.16 & 46 & 0.98 & 1 & 0.02 & 1 & 0.02 \\
\hline $8-2$ & $1367 \mathrm{G}>\mathrm{A} ; \mathrm{R} 456 \mathrm{H}$ & $\mathrm{G}$ & & $A$ & & & $G / G$ & & $\mathrm{G} / \mathrm{A}$ & & $A / A$ & \\
\hline & C, $n=49$ & 91 & 0.93 & 7 & 0.07 & & 42 & 0.86 & 7 & 0.14 & 0 & 0 \\
\hline & $\mathrm{DM}+\mathrm{F}, n=23$ & 41 & 0.89 & 5 & 0.11 & 1.58 & 18 & 0.78 & 5 & 0.22 & 0 & 0 \\
\hline & $\mathrm{DM}, n=32$ & 53 & 0.83 & 11 & 0.17 & 2.70 & 21 & 0.66 & 11 & 0.34 & 0 & 0 \\
\hline & $\mathrm{F}, n=47$ & 87 & 0.93 & 7 & 0.07 & 1.05 & 41 & 0.87 & 5 & 0.11 & 1 & 0.02 \\
\hline & $\mathrm{F}, n=44$ & 81 & 0.92 & 7 & 0.08 & 0.83 & 37 & 0.84 & 7 & 0.16 & 0 & 0 \\
\hline $8-4$ & $1832 \mathrm{~A}>\mathrm{G} ; \mathrm{H} 611 \mathrm{R}$ & $A$ & & $G$ & & & $\mathrm{~A} / \mathrm{A}$ & & $\mathrm{G} / \mathrm{A}$ & & $\mathrm{G} / \mathrm{G}$ & \\
\hline & C, $n=48 t$ & 57 & 0.59 & 39 & 0.41 & & 14 & 0.30 & 29 & 0.60 & 5 & 0.10 \\
\hline & $\mathrm{DM}+\mathrm{F}, n=23^{* *}$ & 43 & 0.93 & 3 & 0.07 & 0.10 & 20 & 0.87 & 3 & 0.13 & 0 & 0 \\
\hline & DM, $n=33$ & 33 & 0.50 & 33 & 0.50 & 1.93 & 11 & 0.33 & 11 & 0.33 & 11 & 0.33 \\
\hline & $\mathrm{F}, n=44$ & 59 & 0.67 & 29 & 0.33 & 0.83 & 21 & 0.48 & 17 & 0.38 & 6 & 0.14 \\
\hline $8-6$ & $2322 G>A ; K 774 K$ & $\mathrm{G}$ & & $A$ & & & $\mathrm{G} / \mathrm{G}$ & & $\mathrm{G} / \mathrm{A}$ & & $\mathrm{A} / \mathrm{A}$ & \\
\hline & C, $n=48 t$ & 87 & 0.91 & 9 & 0.09 & & 39 & 0.81 & 9 & 0.19 & 0 & 0 \\
\hline & $\mathrm{DM}+\mathrm{F}, n=23$ & 41 & 0.89 & 5 & 0.11 & 0.10 & 19 & 0.83 & 3 & 0.13 & 1 & 0.04 \\
\hline & DM, $n=26$ & 48 & 0.92 & 4 & 0.08 & 1.46 & 22 & 0.85 & 4 & 0.15 & 0 & 0 \\
\hline & $\mathrm{F}, n=48$ & 85 & 0.89 & 11 & 0.11 & 0.71 & 38 & 0.79 & 9 & 0.19 & 1 & 0.02 \\
\hline $8-6$ & $2433 \mathrm{G}>\mathrm{A} ; \mathrm{K} 811 \mathrm{~K}$ & $G$ & & A & & & $\mathrm{G} / \mathrm{G}$ & & $\mathrm{G} / \mathrm{A}$ & & $\mathrm{A} / \mathrm{A}$ & \\
\hline & C, $n=48 t$ & 32 & 0.33 & 64 & 0.67 & & 4 & 0.08 & 24 & 0.50 & 20 & 0.42 \\
\hline & $\mathrm{DM}+\mathrm{F}, n=23^{* *}$ & 32 & 0.70 & 14 & 0.30 & 0.22 & 11 & 0.48 & 10 & 043 & 2 & 0.09 \\
\hline & $\mathrm{DM}, n=26^{*}$ & 27 & 0.52 & 25 & 0.48 & 0.46 & 7 & 0.27 & 13 & 0.50 & 6 & 0.23 \\
\hline & $\mathrm{F}, n=48$ & 34 & 0.35 & 62 & 0.65 & 0.88 & 6 & 0.12 & 22 & 0.46 & 20 & 0.42 \\
\hline $8-6$ & $2438 \mathrm{~T}>\mathrm{C} ; \mathrm{V} 813 \mathrm{~A}$ & $\mathrm{~T}$ & & $\mathrm{C}$ & & & $\mathrm{T} / \mathrm{T}$ & & $\mathrm{C} / \mathrm{T}$ & & $\mathrm{C} / \mathrm{C}$ & \\
\hline & C, $n=48 t$ & 95 & 0.99 & 1 & 0.01 & & 47 & 0.98 & 1 & 0.02 & 0 & 0 \\
\hline & $\mathrm{DM}+\mathrm{F}, n=23$ & 46 & 1 & 0 & 0 & 0.68 & 23 & 1 & 0 & 0 & 0 & 0 \\
\hline & DM, $n=26$ & 52 & 1 & 0 & 0 & 0.61 & 26 & 1 & 0 & 0 & 0 & 0 \\
\hline & $\mathrm{F}, n=48$ & 95 & 0.99 & 1 & 0.01 & 1.00 & 47 & 0.98 & 1 & 0.02 & 0 & 0 \\
\hline
\end{tabular}


Table 1 (Continued)

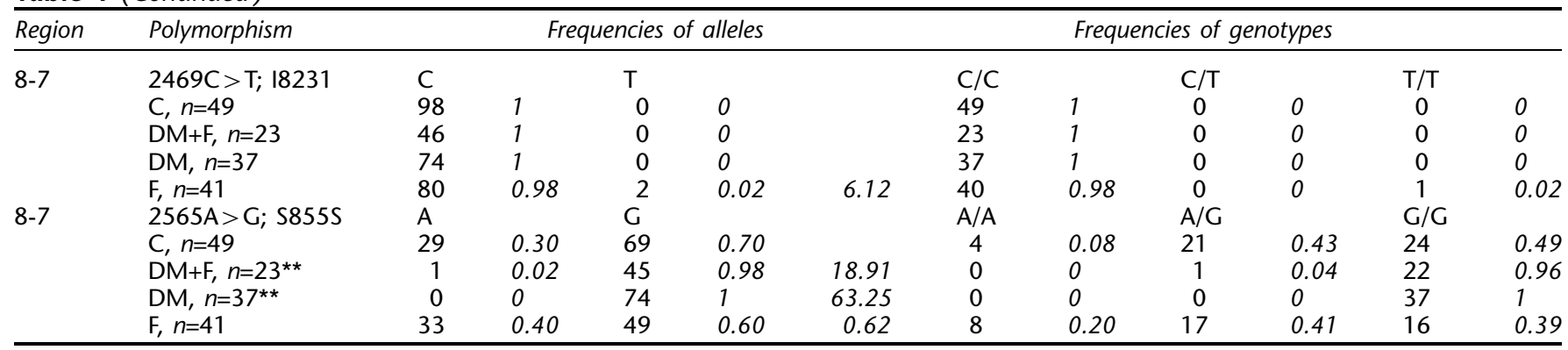

Previously undescribed polymorphisms are shown in italic. $n=$ number of individuals analysed. ${ }^{*}=P<0.05,{ }^{* *}=P<0.0001 . \star$ indicates when $P C R$ amplification was not possible in all samples.

of individuals analysed, we have found significant statistical differences in the distribution of substitutions $1185 \mathrm{~T}>\mathrm{C}$, $1832 \mathrm{~A}>\mathrm{G}$, previously related with type 1 diabetes mellitus, $^{12}$ and in the distribution of the changes $2433 \mathrm{G}>\mathrm{A}$ and $2565 \mathrm{~A}>\mathrm{G}$, in the diabetic groups (DM and/or DM+F) (Table 1). Previous works described the polymorphisms $\mathrm{R} 456 \mathrm{H}, \mathrm{H} 611 \mathrm{R}$ and I720V in WFS1 gene, related with non-autoimmune type 1 diabetes mellitus. ${ }^{12}$ We have observed significant statistical differences in the allelic and genotypic distribution in H611R change in patients with type 2 diabetes mellitus, when compared with the control group. This suggest a possible contribution of this variant with diabetes mellitus.

Three of the four new polymorphisms (T436T, Y455Y, and V831A), as well as mutation V871M, were found in the $\mathrm{F}$ group. This fact suggests that other possible variants could exist in the WFS1 gene, which would contribute to the pathogenic phenotype. Association between deafness and some markers in heterozygosis in the WFS1 gene was previously described. ${ }^{11,13,14}$ In this work, we have not found such a correlation but, perhaps, the analysis of a larger number of deaf patients could reveal association. Unfortunately, we have not been able to perform an association study due to the reduced number of patients available.

We cannot assure whether there is a direct contribution of those alleles with significant statistical differences between affected and control groups in any phenotype.

In spite of all the as yet unclear genetic aspects on WS, we can postulate that missense polymorphisms described in the literature with significant statistical differences in their allelic and genotypic distribution may modulate, directly or indirectly, the normal function of the protein encoded by the WFS1 gene.

In this work, we report a total of five new nucleotide changes and significant statistical differences in distribution of some polymorphisms in a relatively small cohort of patients for each disease. In view of our results and in agreement with previous works, we think that the analysis of the WFS1 gene should be considered in the cases of diabetes mellitus and deafness in which the genetic origin is unknown.

\section{Acknowledgements}

This work was supported by the MCYT (SAF99-0079). E Domènech has an FPI fellowship by the MCYT. Authors thank E Villen and Dr $V$ Volpini for her help in statistical management of data. We also thank the Deafness Research Group of I.R.O. and to Dr J Vendrell and $\mathrm{Dr} F$ Biernès for the samples and comments provided. We acknowledge $H$ Kruyer for revising the manuscript.

\section{References}

1 Wolfram DJ, Wagener HP: Diabetes mellitus and simple optic atrophy among siblings: Report of four cases. Mayo Clin Proc 1938; 13: $715-718$.

2 Barrett TG, Bundey SE: Wolfram (DIDMOAD) syndrome. J Med Genet 1997; 34: $838-841$.

3 Swift RG, Polymeropoulos MH, Torres R et al: Predisposition of Wolfram syndrome heterozygotes to psychiatric illness. Mol Psychiatry 1998; 3: 86-91.

4 Khanim F, Kirk J, Latif F, Barret T: WFS1/wolframin mutations, Wolfram syndrome, and associated diseases. Hum Mutat 2001; 17: $357-367$.

5 Polymeropoulos MH, Swift RG, Swift M: Linkage of the gene for Wolfram syndrome to markers on the short arm of chromosome 4. Nat Genet 1994; 8: 95-97.

6 Inoue $\mathrm{H}$, Tanizawa $\mathrm{Y}$, Wasson $\mathrm{J}$ et al: A gene encoding a transmembrane protein is mutated in patients with diabetes mellitus and optic atrophy (Wolfram syndrome). Nat Genet 1998; 20: 143-148.

7 Strom TM, Hörtnagel K, Hofmann S et al: Diabetes insipidus, diabetes mellitus, optic atrophy and deafness (DIDMOAD) caused by mutations in a novel gene (wolframin) coding for a predicted transmembrane protein. Hum Mol Genet 1998; 13: 2021 - 2028.

8 Takeda K, Inoue H, Tanizawa Y et al: WFS1 (Wolfram syndrome 1) gene product: predominant subcellular localization to endoplasmic reticulum in cultured cells and neuronal expression in rat brain. Hum Mol Genet 2001; 10: 477-484.

9 El-Shanti H, Lidral AC, Jarrah N, Druhan L, Ajlouni K: Homozygosity mapping identifies an additional locus for Wolfram syndrome on chromosome 4q. Am J Hum Genet 2000; 66: 1229-1236.

10 Al-Sheyyab M, Jarrah N, Younis E et al: Bleeding tendency in Wolfram syndrome: a newly identified feature with phenotype genotype correlation. Eur J Pediatr 2001; 160: 243-246. 
11 Young T-L, Ives E, Lynch E et al: Non-syndromic progressive hearing loss DFNA38 is caused by heterozygous missense mutation in the Wolfram syndrome gene. WFS1. Hum Mol Genet 2001; 22: $2509-2514$

12 Awata T, Inoue K, Kurihara S et al: Missense variations of the gene responsible for Wolfram syndrome (WFS1/wolframin) in Japanese: possible contribution of the Arg456His mutation to type 1 diabetes as a nonautoimmune genetic basis. Biochem Biophys Res Commun 2000; 268: 612-616.
13 Ohata T, Koizumi A, Kayo T et al: Evidence of an increased risk of hearing loss in heterozygous carriers in a Wolfram syndrome family. Hum Genet 1998; 103: 470-474.

14 Bespalova I, Van Camp G, Bom S JH et al: Mutations in the Wolfram syndrome 1 gene (WFS1) are a common cause of low frequency sensorineural hearing loss. Hum Mol Genet 2001; 22: $2501-2508$. 